\title{
The energy dependence of the diffraction minimum in the elastic scattering and new LHC data
}

\author{
O. V. Selyugin \\ BLTPh, JINR, Dubna, Russia
}

\begin{abstract}
The soft diffraction phenomena in the elastic proton-proton scattering are reviewed from the viewpoint of experiments at the LHC (TOTEM and ATLAS collaboration). In the framework of the High Energy Generalized Structure (HEGS) model the form of the diffraction minimum in the nucleon-nucleon elastic scattering in a wide energy region is analyzed. The energy dependencies of the main characteristics of the diffraction dip are obtained. The numerical predictions at LHC energies are presented. The comparison of the model predictions with the new LHC data at $\sqrt{s}=13 \mathrm{TeV}$ is made.
\end{abstract}

Keywords: hadrons, high energies, eikonal, diffraction dip, LHC data

\section{Introduction}

A great amount of experimental and theoretical researches of high energy elastic proton-proton and proton-antiproton scattering in a wide region of the momentum transfer provide reach information on these processes [1], which allows us to narrow the circle of examined models and at the same time to set a number of difficult problems, which are not yet solved, concerning mainly the energy dependence of characteristics of these reactions.

It is just this process that allows the verification of the results obtained from the main principles of quantum field theory: the concept of the scattering amplitude as a unified analytic function of its kinematic variables connecting different reaction channels introduced in the dispersion theory by N.N. Bogoliubov. The recent results obtained at the collider accelerators and in the cosmic experiments show a still continuing growth of the total cross sections, the diffraction peak shrinkage and a slow growth of the relation of the elastic to the total cross sections. A especial question is about the 
behavior of the phase of the elastic scattering amplitude, which can be presented in the form of the ratio of the real to imaginary part of the scattering amplitude $-\rho(s, t)=\operatorname{Re} F(s, t) / \operatorname{Im} F(s, t)$, which is tightly connected with the dispersion relations. Also, the question about the energy dependence of the spin-flip amplitude has to be noted. In most of the early models, as in the ordinary picture of perturbative quantum chromodynamics (PQCD), the spin effects were suppressed at large energies. However, in some models $[2,3,44,5,6]$ the spin-flip amplitudes, which are related with some different nonperturbative processes not decreasing or slowly decreasing with growing energy, were predicted.

The recent results from the LHC pose new questions in the study of the structure of hadronic amplitudes, as its give the important information about the soft hadron processes at super high energies. The new data of the TOTEM and ATLAS Collaborations indeed show that none of the models predicted correctly the elastic cross sections at the LHC.

One of the main problems of the dynamical models is linked to the structure of hadrons which should be presented by the conventional electromagnetic form factors of the hadrons, or via Generalized Parton Distributions (GPDs), under the assumption that hadrons respond to Pomerons in the same way as they do to photon. In practice, many models took into account these assumptions and used some phenomenological forms of the form factors with the extra parameters determined by a fit of the experimental data. For example in [7] purely phenomenological exponential form factors are used. Obviously, such exponential form cannot be used at sufficiently large momentum transfer, as does not correspond to the power dependence of the form factors which is require the quark model.

In papers [8, 9], the dynamical model for a hadron interaction, which takes into account the hadron structure at large distances through the generalized parton distribution functions, was developed to describe quantitatively and simultaneously the proton-proton and proton antiproton elastic scattering at high energies. The model is based on the general quantum field theory principles (analyticity, unitarity and so on) and takes into account the basic information on the structure of a nucleon as a compound system.

The measure of the $s$-dependence of the total cross sections $\sigma_{\text {tot }}(s)$ and of $\rho(s, t)$ - the ratio of the real to imaginary part of the elastic scattering amplitude, is very important as they are connected to each other through the integral dispersion relations. The validity of this relation can be checked at LHC energies The deviation can point out the existence of a fundamental 
length at $\mathrm{TeV}$ energies [10, 11]. However, for such a conclusion we should know with high accuracy the lower energies data as well.

As we do not know exactly, from a theoretical viewpoint, the dependence of the scattering amplitude on $s$ and $t$, it is usually assumed that the imaginary and real parts of the spin-non-flip amplitude behave exponentially with the same slope. Similarly, one assumes the imaginary and real parts of the spin-flip amplitudes (without the kinematic factor $\sqrt{|t|}$ ) to have an analogous $t$-dependence in the examined domain of momenta transfer. Moreover, one assumes energy independence of the ratio of spin-flip to spin-non-flip parts at small $t$. All this is our theoretical uncertainty.

Of course, we have plenty of experimental data in the domain of small $t$ at low energies $3<p_{L}<100(\mathrm{GeV} / \mathrm{c})$. Unfortunately, most of these data come with large errors. The extracted sizes of $\rho(s, t=0)$ contradict each other in the different experiments and give a bad $\chi^{2}$ in the different models trying to describe the $s$-dependence of $\rho(s, t=0)$ (see, for example, the results of the COMPETE Collaboration [12, 13]. It is of first importance that a more careful analysis of these experimental data gives in some cases an essentially different extrapolation for $\rho(s, t=0)$. For example, the analysis of the experimental data made in [14], which takes into account the uncertainty of the total cross sections (3-parameters fit) and the uncertainty of the Luminosity (4-parameters fit) gave a $\rho(s, t=0)$, which differs from the original values obtained by the experimental group, by $25 \%$ on average. For example, for $p_{L}=19.23 \mathrm{GeV} / \mathrm{c}$ the experimental work gave $\rho(s, t=0)=-0.25 \pm 0.03$ and for $p_{L}=38.01 \mathrm{GeV} / \mathrm{c} \rho(s, t=0)=-0.17 \pm 0.03$. The analysis with free 4-parameters gave for these values: $\rho(s, t=0)=-0.32 \pm 0.08$ and $\rho(s, t=0)=-0.12 \pm 0.03$, respectively. This kind of picture was confirmed by the independent analysis of the experimental data [15, 16] $52<p_{L}<400(\mathrm{GeV} / \mathrm{c})$ of Fajardo [17] and Selyugin [14]. Both new analysis coincide with each other but differ from the original experimental determination.

The non-trivial procedure of the extraction of the size of $\rho(s, t)$ from the experimental data on the differential cross sections shows the semiphenomenological properties of $\rho(s, t)$ [18]. Its size is dependent on some theoretical assumption [19]. For example, a significant discrepancy in the experimental measurement of $\rho$ was found by the UA4 and UA4/2 collaborations at $\sqrt{s}=541 \mathrm{GeV}$. But a more careful extrapolation [20] to $t=0$ shows that there is no real contradiction between these measurements and gives for this energy $\rho(\sqrt{s}=541 \mathrm{GeV}, t=0)=0.163$, the same as in the 
previous phenomenological analysis [14].

In this paper, we consider in detail the situation in the region of the diffraction dip where the real part (and possibly spin-flip amplitude) plays the essential role. The proposed model takes into account all known features of the near forward proton-proton and proton-antiproton data, the properties of the spin-non-flip and spin-flip amplitudes, total cross sections, ratios of the real to the imaginary forward amplitudes and Coulomb-nuclear interference phase where the form factors of the nucleons are also taken into account.

\section{The elastic nucleon scattering in the framework of the HEGS model}

The differential cross sections of nucleon-nucleon elastic scattering can be written as the sum of different helicity amplitudes:

$$
\frac{d \sigma}{d t}=\frac{2 \pi}{s^{2}}\left(\left|\phi_{1}\right|^{2}+\left|\phi_{2}\right|^{2}+\left|\phi_{3}\right|^{2}+\left|\phi_{4}\right|^{2}+4\left|\phi_{5}\right|^{2}\right) .
$$

The HEGS model [8, 9] takes into account all five spiral electromagnetic amplitudes. The electromagnetic amplitude can be calculated in the framework

of QED. In the high energy approximation, it can be obtained [21] for the spin-non-flip amplitudes:

$$
F_{1}^{e m}(t)=\alpha_{e m} f_{1}^{2}(t) \frac{s-2 m^{2}}{t} ; \quad F_{3}^{e m}(t)=F_{1}^{e m}
$$

where $\alpha_{e m}$ is the electromagnetic fine-structure constant, and for the spin-flip amplitudes:

$F_{2}^{e m}(t)=\alpha_{e m} \frac{f_{2}^{2}(t)}{4 m^{2}} s ; \quad F_{4}^{e m}(t)=-F_{2}^{e m}(t), \quad F_{5}^{e m}(t)=\alpha_{e m} \frac{s}{2 m \sqrt{|t|}} f_{1}(t) f_{2}(t)$

where the form factors are:

$$
f_{1}(t)=\frac{4 m_{p}^{2}-(1+k) t}{4 m_{p}^{2}-t} G_{d}(t) ; \quad f_{2}(t)=\frac{4 m_{p}^{2} k}{4 m_{p}^{2}-t} G_{d}(t)
$$

with $k$ relative to the anomalous magnetic moment, and $G_{d}(t)$ has the conventional dipole form $G_{d}(t)=1 /(1-t / 0.71)^{2}$. With the electromagnetic and hadronic interactions included, every amplitude $\phi_{i}(s, t)$ can be described as

$$
\phi_{i}(s, t)=F_{i}^{e m} \exp \left(i \alpha_{e m} \varphi(s, t)\right)+F_{i}^{h}(s, t),
$$


where $\varphi(s, t)=\varphi_{C}(t)-\varphi_{C h}(s, t)$, and $\varphi_{C}(t)$ will be calculated in the second Born approximation in order to allow the evaluation of the Coulomb-hadron interference term $\varphi_{C h}(s, t)$. The quantity $\varphi(s, t)$ has been calculated and discussed by many authors (see [22] and references therein).

Let us define the hadronic spin-non-flip amplitudes as

$$
F_{\mathrm{nf}}^{h}(s, t)=\left[F_{1}^{h}(s, t)+F_{3}^{h}(s, t)\right] / 2 ;
$$

The model is based on the representation that at high energies a hadron interaction in the non-perturbative regime is determined by the reggeizedgluon exchange. The cross-even part of this amplitude can have two nonperturbative parts, possible standard pomeron $\left(P_{2 n p}\right)$ and cross-even part of the 3-non-perturbative gluons $\left(P_{3 n p}\right)$. The interaction of these two objects is proportional to two different form factors of the hadron. This is the main assumption of the model. The second important assumption is that we chose the slope of the second term four times smaller than the slope of the first term, by analogy with the two pomeron cut. Both terms have the same intercept.

The parton picture of hadron structure is represented, in most part, by the parton distribution functions (PDFs). They are determined in deep inelastic processes. The next step in the development of the picture of hadron structure was made by introducing the non-forward structure functions - general parton distributions - GPDs with the spin-independent $H(x, \xi, t)$ and the spin-dependent $E(x, \xi, t)$ parts. Some of the advantages of GPDs were presented by the sum rules [23]. Using the different momenta of GPDs as a function of $x^{n-1}$ we can obtain the different form factors Compton form factors: $R_{V}(t), R_{A}(t), R_{T}(t)$; the electromagnetic form factors $F_{1}(t), F_{2}(t)$ and the gravimagnetic form factors $A_{1}(t), B_{2}(t)$. In [25] different PDFs sets were examined and the momentum transfer dependence of the GPDs $H(x, t, \xi=0)$ and $E(x, t, \xi=0)$ was obtained which give the best descriptions of the electromagnetic form factors of the proton and neutron simultaneously. It allows us to calculate two different form factors: the electromagnetic form factors can be represented as the first moments of GPDs $H(x, t)$ and $E(x, t)$, and the integration of the second moment of GPDs over $x$ gives the momentumtransfer representation of the form factor $A(t)$, which is reflects the matter distribution in the hadron (see [8, 9, 25]).

The parameters and $t$-dependence of the GPDs are determined by the standard parton distribution functions, so by the experimental data on the 
deep inelastic scattering, and by the experimental data for the electromagnetic form factors (see [24] ). The calculations of the form factors were carried out in [25].

Hence, the Born term of the elastic hadron amplitude can now be written as

$$
\begin{aligned}
F_{h}^{\text {Born }}(s, t)=\quad & h_{1} G^{2}(t) F_{a}(s, t)\left(1+r_{1} / \hat{s}^{0.5}\right)+h_{2} A^{2}(t) F_{b}(s, t) \\
& \pm h_{\text {odd }} A^{2}(t) F_{b}(s, t)\left(1+r_{2} / \hat{s}^{0.5}\right),
\end{aligned}
$$

where the last term represents the Odderon contribution and the upper sign is related to $p \bar{p}$ and the lower sign to $p p . F_{a}(s, t)$ and $F_{b}(s, t)$ have the standard Regge form: $F_{a}(s, t)=\hat{s}^{\epsilon_{1}} e^{B(\hat{s}) t} ; F_{b}(s, t)=\hat{s}^{\epsilon_{1}} e^{B(\hat{s}) / 4 t}, \hat{s}=s e^{-i \pi / 2} / s_{0}$ $; s_{0}=4 m_{p}^{2} \mathrm{GeV}^{2}$, and $h_{\text {odd }}=i h_{3} t /\left(1-r_{0}^{2} t\right)$. The slope of the scattering amplitude has the standard logarithmic dependence on the energy $B(s)=$ $\alpha^{\prime} \ln (\hat{s})$ with $\alpha^{\prime}=0.24 \mathrm{GeV}^{-2}$ and with some small additional term [9] which reflect the small non-linear behavior of $\alpha^{\prime}$ at small momentum transfer [26]. The final elastic hadron scattering amplitude is obtained after unitarization of the Born term. So, at first, we have to calculate the eikonal phase

$$
\chi(s, b)=-\frac{1}{2 \pi} \int d^{2} q e^{i \vec{b} \cdot \vec{q}} F_{h}^{\text {Born }}\left(s, q^{2}\right),
$$

where $q^{2}=-t$, and then obtain the final hadron scattering amplitude

$$
F_{h}(s, t)=i s \int b J_{0}(b q) \Gamma(s, b) d b \quad \Gamma(s, b)=1-\exp [\chi(s, b)] .
$$

At large $t$ our model calculations are extended up to $-t=15 \mathrm{GeV}^{2}$. We added a small contribution of the energy independent part of the spin flip amplitude in the form similar to the proposed in [27] and analyzed in [28].

$$
F_{s f}(s, t)=h_{s f} q^{3} F_{1}^{2}(t) e^{-B_{s f} q^{2}} .
$$

The model is very simple from the viewpoint of the number of fitting parameters and functions. There are no any artificial functions or any cuts which bound the separate parts of the amplitude by some region of momentum transfer.

3416 experimental points were included in our analysis in the energy region $9.8 \mathrm{GeV} \leq \sqrt{s} \leq 8$. TeV and in the region of momentum transfer $0.000375 \leq|t| \leq 15 \mathrm{GeV}^{2}$. The experimental data of the proton-proton and 
proton-antiproton elastic scattering are included in 92 separate sets of 32 experiments [29, 30] including recent data of the TOTEM Collaboration at $\sqrt{s}=8 \mathrm{TeV}$ [31]. The whole Coulomb-hadron interference region, where the experimental errors are remarkably small, was included in our examination of the experimental data. As the result, it was obtained $\sum_{i=1}^{N} \chi_{i}^{2} / N=1.28$ with the parameters $h_{1}=3.66 ; \quad h_{2}=1.39 ; \quad h_{3}=0.76 ; \quad k_{0}=0.16 ; \quad r_{0}^{2}=3.82$;

and the low energy parameters $h_{s f}=0.05 ; \quad R_{1}=53.7 ; \quad R_{2}=4.45$.

Such a simple form of the scattering amplitude in the huge region of energy requires careful determination of the slope of the scattering amplitude. In the present model, a small additional term is introduced into the slope, which reflects some possible small nonlinear properties of the intercept and leads to the standard form of the slope as $t \rightarrow 0$ and $t \rightarrow \infty[9]$.

\section{The differential cross sections at LHC energies}

Let us see the predictions of the HEGS model for the LHC energies. The result of the calculations of the differential cross sections of the elastic proton-proton scattering at $\sqrt{s}=8 \mathrm{TeV}$ and $\sqrt{s}=13 \mathrm{TeV}$ are presented in Table 1 and Table 2. In the model, the data on the total cross section are not included in the fitting procedure as its value is extracted from the corresponding differential cross sections by one or another procedure. Hence, the sizes of $\sigma_{t o t}(s)$ are obtained in the model through the optic theorem by the calculation of the imaginary part of the hadronic amplitude at zero value of the momentum transfer. The corresponding values of the obtained total cross sections show a good coincidence with the experimental data [9, 26] in the wide energy region.

The arithmetic mean on the value of the total cross sections obtained by the different methods by the TOTEM Collaboration at $7 \mathrm{TeV}$ is $98.5 \pm 2.9$ $\mathrm{mb}$ and at $\sqrt{s}=8 \mathrm{TeV}-102.9 \pm 2.3 \mathrm{mb}$. The ATLAS Collaboration for these energies give the value of the $\sigma_{t o t}=95.35 \pm 2.3$ and $96.07 \pm 1.34 \mathrm{mb}$. Obviously, there is a large difference between the data of the TOTEM and ATLAS Collaborations which grows at $\sqrt{s}=8 \mathrm{TeV}$.

Let us see the experimental data of the differential cross sections in the small momentum transfer region at LHC energies. Now there are five sets of experimental data on the elastic $p p$ scattering at LHC energies and small momentum transfer: it is the data of the TOTEM Collaborations at $7 \mathrm{TeV}$ [32, 33], at $8 \mathrm{TeV}$ [34], and the data of the ATLAS Collaborations at 7 
Table 1: $p p$ scattering (predictions of the HEGS model at $\sqrt{s}=8 \mathrm{TeV}$ )

\begin{tabular}{|c|c|c|c|c|c|}
\hline $\begin{array}{c}-t \\
{\left[\mathrm{GeV}^{2}\right]} \\
0.0005\end{array}$ & $\begin{array}{c}d \sigma_{e l} / d t \\
{\left[\mathrm{mb} / \mathrm{GeV}^{2}\right]} \\
1317.00\end{array}$ & $\begin{array}{c}-t \\
{\left[G e V^{2}\right]} \\
0.0900\end{array}$ & $\begin{array}{c}d \sigma_{e l} / d t \\
{\left[m b / G e V^{2}\right]} \\
81.7700\end{array}$ & $\begin{array}{c}-t \\
{\left[G e V^{2}\right]} \\
0.50\end{array}$ & $\begin{array}{c}d \sigma_{e l} / d t * 10^{3} \\
{\left[m b / G e V^{2}\right]} \\
15.7300\end{array}$ \\
\hline 0.0010 & 650.80 & 0.0950 & 73.9800 & 0.51 & 15.9080 \\
\hline 0.0020 & 501.20 & 0.1100 & 54.7600 & 0.52 & 16.3800 \\
\hline 0.0030 & 472.20 & 0.1200 & 44.8000 & 0.53 & 17.0300 \\
\hline 0.0040 & 458.00 & 0.1300 & 36.6200 & 0.54 & 17.8000 \\
\hline 0.0050 & 447.40 & 0.1400 & 29.9300 & 0.55 & 18.6200 \\
\hline 0.0060 & 438.00 & 0.1600 & 19.9700 & 0.56 & 19.4400 \\
\hline 0.0070 & 429.30 & 0.1800 & 13.2900 & 0.57 & 20.2400 \\
\hline 0.0080 & 420.80 & 0.2000 & 8.8100 & 0.58 & 21.0000 \\
\hline 0.0100 & 404.50 & 0.2200 & 5.8180 & 0.60 & 22.2000 \\
\hline 0.0120 & 388.80 & 0.2400 & 3.8210 & 0.64 & 23.4000 \\
\hline 0.0140 & 373.60 & 0.2600 & 2.2491 & 0.70 & 22.8000 \\
\hline 0.0160 & 359.00 & 0.2800 & 1.6090 & 0.80 & 18.9000 \\
\hline 0.0180 & 344.90 & 0.3000 & 1.0280 & 0.90 & 13.2200 \\
\hline 0.0200 & 331.40 & 0.3200 & 0.6485 & 1.00 & 9.0000 \\
\hline 0.0220 & 318.40 & 0.3400 & 0.4029 & 1.10 & 5.9570 \\
\hline 0.0240 & 306.00 & 0.3600 & 0.2457 & 1.20 & 3.8910 \\
\hline 0.0270 & 288.20 & 0.3700 & 0.1906 & 1.30 & 2.5300 \\
\hline 0.0300 & 271.50 & 0.3800 & 0.1472 & 1.40 & 1.6400 \\
\hline 0.0350 & 245.70 & 0.3900 & 0.1133 & 1.50 & 1.0600 \\
\hline 0.0400 & 222.40 & 0.4000 & 0.0870 & 1.60 & 0.6870 \\
\hline 0.0450 & 201.20 & 0.4100 & 0.0668 & 1.7 & 0.4440 \\
\hline 0.0500 & 182.10 & 0.4200 & 0.0515 & 1.8 & 0.2833 \\
\hline 0.0550 & 164.70 & 0.4300 & 0.0400 & 1.9 & 0.1820 \\
\hline 0.0600 & 149.10 & 0.4400 & 0.0315 & 2.0 & 0.1150 \\
\hline 0.0650 & 134.90 & 0.4500 & 0.0254 & 2.1 & 0.0730 \\
\hline 0.0700 & 122.10 & 0.4600 & 0.0212 & 2.2 & 0.0460 \\
\hline 0.0750 & 110.50 & 0.4700 & 0.0185 & 2.3 & 0.0290 \\
\hline 0.0800 & 99.90 & 0.4800 & 0.0168 & 2.4 & 0.0180 \\
\hline 0.0850 & 90.40 & 0.4900 & 0.0160 & 2.5 & 0.0120 \\
\hline
\end{tabular}


Table 2: $p p$ scattering (predictions of the HEGS model at $\sqrt{s}=13 \mathrm{TeV}$ )

\begin{tabular}{|c|c|c|c|c|c|}
\hline $\begin{array}{c}-t \\
{\left[G e V^{2}\right]}\end{array}$ & $\begin{array}{c}d \sigma_{e l} / d t \\
{\left[m b / G e V^{2}\right]}\end{array}$ & $\begin{array}{c}-t \\
{\left[G e V^{2}\right]}\end{array}$ & $\begin{array}{c}d \sigma_{e l} / d t \\
{\left[m b / G e V^{2}\right]}\end{array}$ & $\begin{array}{c}-t \\
{\left[G e V^{2}\right]}\end{array}$ & $\begin{array}{r}d \sigma_{e l} / d t * 10^{3} \\
{\left[m b / G e V^{2}\right]}\end{array}$ \\
\hline 0.0005 & 1389.00 & 0.0900 & 87.5700 & 0.50 & 30.400 \\
\hline 0.0010 & 728.71 & 0.0950 & 78.7700 & 0.51 & 32.0000 \\
\hline 0.0020 & 579.90 & 0.1100 & 57.3100 & 0.52 & 33.5000 \\
\hline 0.0030 & 549.70 & 0.1200 & 46.3100 & 0.53 & 34.8000 \\
\hline 0.0040 & 533.70 & 0.1300 & 37.4000 & 0.54 & 36.0000 \\
\hline 0.0050 & 521.30 & 0.1400 & 30.1700 & 0.55 & 37.0000 \\
\hline 0.0060 & 510.10 & 0.1600 & 19.5900 & 0.56 & 37.9000 \\
\hline 0.0070 & 499.50 & 0.1800 & 12.6500 & 0.57 & 38.5000 \\
\hline 0.0080 & 489.20 & 0.2000 & 8.1200 & 0.58 & 39.0000 \\
\hline 0.0100 & 469.40 & 0.2200 & 5.1710 & 0.60 & 39.4000 \\
\hline 0.0120 & 450.30 & 0.2400 & 3.2620 & 0.64 & 38.3000 \\
\hline 0.0140 & 432.00 & 0.2600 & 2.0320 & 0.70 & 33.9000 \\
\hline 0.0160 & 414.30 & 0.2800 & 1.2500 & 0.80 & 24.3000 \\
\hline 0.0180 & 397.30 & 0.3000 & 0.7500 & 0.90 & 16.1000 \\
\hline 0.0200 & 381.50 & 0.3200 & 0.4430 & 1.00 & 10.3000 \\
\hline 0.0220 & 365.50 & 0.3400 & 0.2550 & 1.10 & 6.4400 \\
\hline 0.0240 & 350.50 & 0.3600 & 0.1440 & 1.20 & 4.0000 \\
\hline 0.0270 & 329.20 & 0.3700 & 0.1080 & 1.30 & 2.5000 \\
\hline 0.0300 & 309.30 & 0.3800 & 0.0806 & 1.40 & 1.5400 \\
\hline 0.0350 & 278.60 & 0.3900 & 0.0609 & 1.50 & 0.9500 \\
\hline 0.0400 & 250.90 & 0.4000 & 0.0470 & 1.60 & 0.5900 \\
\hline 0.0450 & 225.90 & 0.4100 & 0.0370 & 1.7 & 0.3600 \\
\hline 0.0500 & 203.40 & 0.4200 & 0.0310 & 1.8 & 0.2200 \\
\hline 0.0550 & 183.10 & 0.4300 & 0.0270 & 1.9 & 0.1300 \\
\hline 0.0600 & 164.90 & 0.4400 & 0.0253 & 2.0 & 0.0800 \\
\hline 0.0650 & 148.40 & 0.4500 & 0.0246 & 2.1 & 0.0480 \\
\hline 0.0700 & 133.60 & 0.4600 & 0.0249 & 2.2 & 0.0290 \\
\hline 0.0750 & 120.20 & 0.4700 & 0.0258 & 2.3 & 0.0180 \\
\hline 0.0800 & 108.20 & 0.4800 & 0.0270 & 2.4 & 0.0120 \\
\hline 0.0850 & 97.34 & 0.4900 & 0.0290 & 2.5 & 0.0080 \\
\hline
\end{tabular}



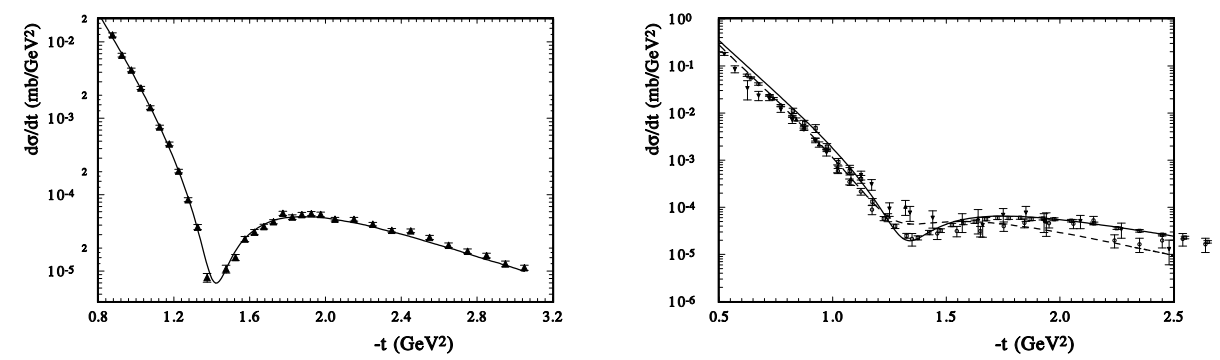

Figure 1: The model calculation of the diffraction minimum in $d \sigma / d t$ of $p p$ scattering [left] at $\sqrt{s}=30.4 \mathrm{GeV}$; [right] for $p p$ and $p \bar{p}$ at $\sqrt{s}=52.8 \mathrm{GeV}$ scattering.

$\mathrm{TeV}$ [35] and at $8 \mathrm{TeV}$ [36]. Recently, there have appeared preliminary nonnormalized data at $13 \mathrm{TeV}$ [37] of the TOTEM Collaboration.

The comparison of the predictions of the HEGS model with these data [26] shows that the main problem of these data is concentrated in the normalization of the differential cross sections. The data of the ATLAS Collaboration practically exactly coincide with the model calculations, and the additional normalization $n$ is really near unity. However, the TOTEM data require additional normalization $n=0.95 ; 0.91$ respectively, at $7 \mathrm{TeV}$ and $8 \mathrm{TeV}$. The data at $13 \mathrm{TeV}$ have no normalization. However, their form coincides with the form of the model predictions sufficiently well.

\section{The dip region}

Now let us examine the form of the differential cross section in the region of the momentum transfer where the diffractive properties of the elastic scattering appear most strongly - it is the region of the diffraction dip. The form and the energy dependence of the diffraction minimum are very sensitive to the different parts of the scattering amplitude. The change of the sign of the imaginary part of the scattering amplitude determines the position of the minimum and its movement with changing the energy. The real part of the scattering amplitude determines the size of the dip. Hence, it depends heavily on the odderon contribution. The spin-flip amplitude gives the contribution to the differential cross sections additively. So the measurement of the form and energy dependence of the diffraction minimum with high preci- 


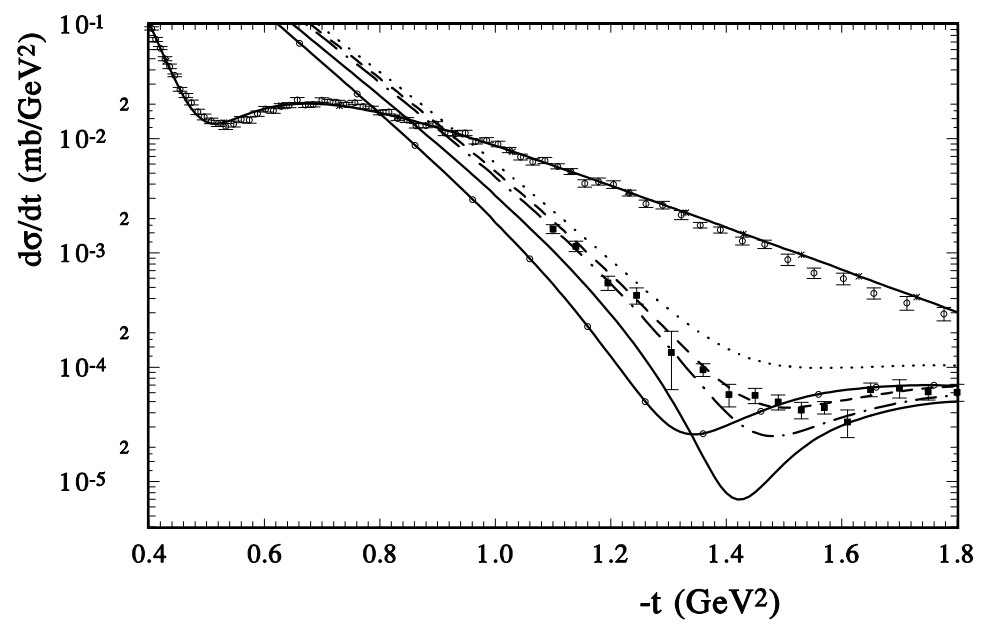

Figure 2: The model calculation of the diffraction minimum in $d \sigma / d t$ of $p p$ at $\sqrt{s}=$ $13.4 ; 16.8 ; 19.4 ; 30.4 ; 52.8 ; 7000 \mathrm{GeV}$; (lines correspondingly - dots; short dash; dot-dash; solid; solid+circles; solid+ants); the squares - the data at $\sqrt{s}=16.82 \mathrm{GeV}$, and the circles - the data at $\sqrt{s}=7 \mathrm{TeV}[32]$.

sion is an important task for future experiments. In Fig.1, the description of the diffraction minimum in our model is shown for ISR energies. The HEGS model reproduces sufficiently well the energy dependence and the form of the diffraction dip. In this energy region the diffraction minimum reaches the sharpest $\operatorname{dip}$ at $\sqrt{s}=30 \mathrm{GeV}$. Note that at this energy the value of $\rho(s, t=0)$ also changes its sign in the proton-proton scattering. The $p \bar{p}$ cross sections in the model are obtained by the $s \rightarrow u$ crossing without changing the model parameters. And for the proton-antiproton scattering the same situation with correlations between the sizes of $\rho(s, t=0)$ and $\rho\left(s, t_{\text {min }}\right)$ takes place at low energy (approximately at $p_{L}=100 \mathrm{GeV}$ ).

The HEGS model reproduces $d \sigma / d t$ at very small and large $t$ and provides a qualitative description of the dip region at $-t \approx 1.6 \mathrm{GeV}^{2}$, for $\sqrt{s}=10 \mathrm{GeV}$ and $-t \approx 0.45 \mathrm{GeV}^{2}$ for $\sqrt{s}=13 \mathrm{TeV}$ (Fig.2). Note that it gives a good description for the proton-proton and proton-antiproton elastic scattering or $\sqrt{s}=53 \mathrm{GeV}$ and for $\sqrt{s}=62.1 \mathrm{GeV}$. The diffraction minimum at $\sqrt{s}=7 \mathrm{TeV}$ and $\sqrt{s}=13 \mathrm{TeV}$ is reproduced sufficiently well too (Fig.3).

The dependence of the position of the diffraction minimum on $t$ is determined in most part by the growth of the total cross sections and the 


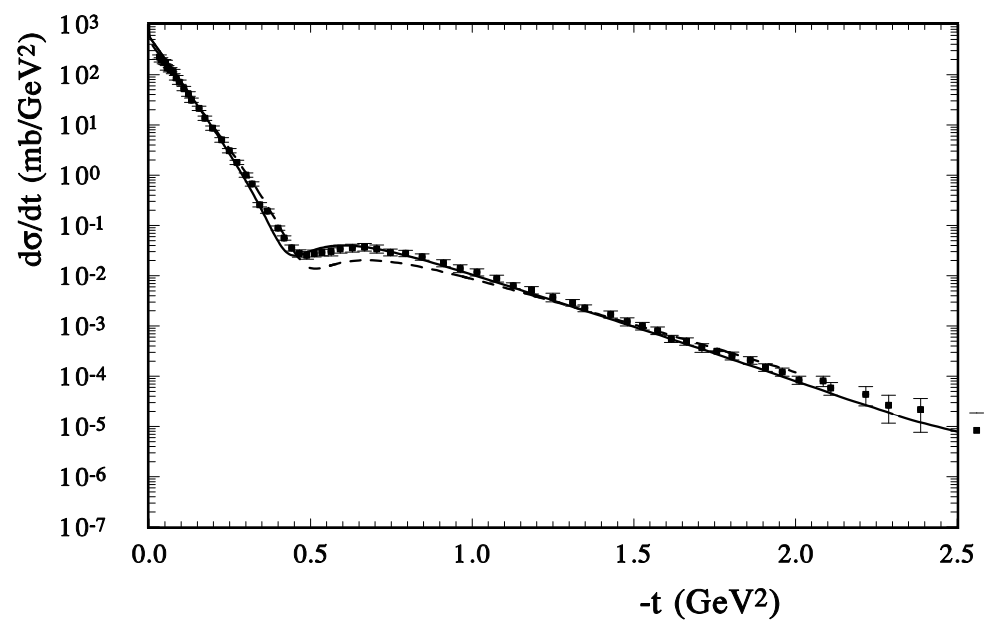

Figure 3: The model predictions of $d \sigma / d t$ (dashed line for $\sqrt{s}=8 \mathrm{TeV}$ (Table 1) and solid line $\sqrt{s}=13 \mathrm{TeV}$ (Table 2); points - the non-normalized experimental data of the TOTEM Collaborations at $\sqrt{s}=13 \mathrm{TeV}$ [37] (normalized on the model calculations).

slope of the imaginary part of the scattering amplitude. In the framework of the geometrical scaling approximation [38] it was proposed in [39] that the basic values of the elastic scattering are proportional to the effective radius of the interaction $\sigma_{t o t}(s) \sim R^{2}(s), \sigma_{e l}(s) \sim R^{2}(s), B(s) \sim R^{2}(s)$, $t_{\text {min }}(s) \sim R^{2}(s)$. In this case, we have $t_{\text {min }} \sigma_{\text {tot }}=$ constant. Now most models use the hypothesis of the factorization eikonal [40] where the eikonal phase $\chi(s, t)=f(s) f_{0}(b / R(s))$. The experimental data in most part support the last hypothesis and the geometrical scaling valid only in some region of the energy on the average.

Figure 2 shows such a dependence obtained in the HEGS model in the huge energy interval. The energy dependence of the position of the diffraction minimum $t_{\min }(s, t)$ can be reproduced by the simple approximation, with a right value as $s \rightarrow \infty$,

$$
-t_{\min }=a_{1} /\left[1+a_{2} \ln \left(s / s_{0}\right)^{n}\right],
$$

where $a_{1}=1.85 \pm 0.08$ and $a_{2}=0.009 \pm 0.001$ are the free parameters and $s_{0}=4 m_{p}^{2}$ (as in the HEGS model), $n=2$ with $\sum \chi_{i}^{2}=0.4$; (the shortdashed line in Fig.4a for the simplest case with two parameters), and the case $a_{1}=1.61 \pm 0.11$ and $a_{2}=0.002 \pm 0.0003$ with $\sum \chi_{i}^{2}=3.8$, if it is taken 

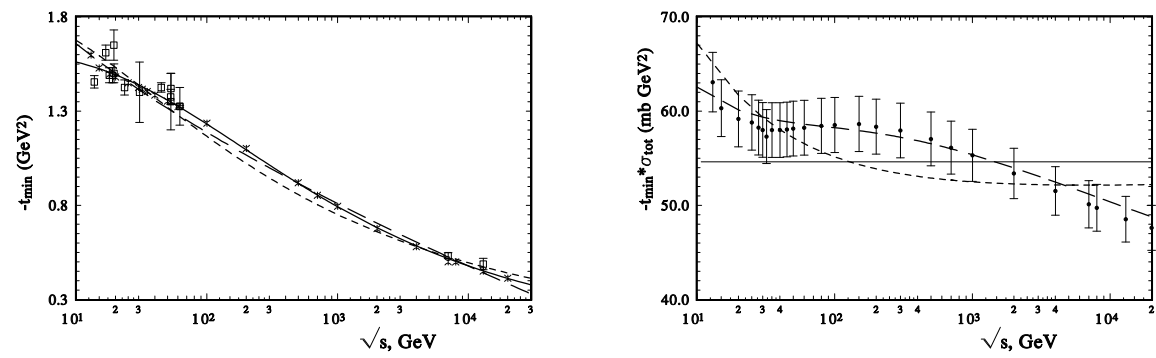

Figure 4: [left] The energy dependence of the position of the diffraction minimum $-t_{\text {min }}$ (ants - the determination of $t_{\text {min }}$ from the HEGS model calculations; squares - the $t_{\text {min }}$ determined from experimental data; the solid and short dashed lines (the approximations by eq.(11) with $n=2.6$ and $n=2.0)$ ); the long dashed line - the approximation by eq. (12); [right] the energy dependence of the product $t_{m i n} \sigma_{t o t}$ (hard line - $C=54.8 \mathrm{mb}$ $\mathrm{GeV}^{2}$, point - the HEGS model calculations, dashed line - the product of $t_{\min }$ (eq.(11), $n=2)$ and $\sigma_{t o t} \operatorname{PDG}([42])$; long-dashed line - the product of $t_{\min }$ (eq.(11), $n=2.6$ ) and $\left.\left.\sigma_{\text {tot }} \operatorname{PDG}(42]\right)\right)$.

into account as a free parameter $n=2.6 \pm 0.35$ (hard line in Fig.4a). In Fig.4a the errors of the data obtained from the model calculations are taken as $1 \%$ from the size of $t_{m i n}$. A good approximation can be obtained else by

$$
-t_{\text {min }}=a_{1}-a_{2} \ln \left(s / s_{0}\right)^{n} \text {. }
$$

In this case $a_{1}=2.28 \pm 0.27$ and $a_{2}=0.11 \pm 0.01$ and $n=0.65 \pm 0.035$ (longdashed line in Fig.4). Despite that this equation gives a better approximation of the energy dependence of $t_{m i n}$, it has a bad asymptotic value as $s \rightarrow \infty$.

In [41], the assumption about the scaling properties of $t_{\text {min }}$ and $\sigma_{\text {tot }}$ was introduced as $t_{\text {min }} \sigma_{t o t} \approx C$ where $C=54.8 \pm 0.7 \mathrm{mb} \mathrm{GeV}$. In comparing the form of eq.(11) with the approximation of the total cross section (for example PDG [42]), such scaling properties are confirmed at first sight. However, the detailed examination shows some difference. The scaling properties are performed on the average, see Fig.4b (hard line) and the product of $t_{\text {min }}$ (eq.(11), $n=2.0$ ) and $\sigma_{\text {tot }} \operatorname{PDG}(42]$ ) (Fig.4b - short-dashed line). Really, the energy dependence of such a product has a complicated form. The calculations in the framework of the HEGS model and with and energy independence of the product of $t_{\min }$ (eq.(11) with $n=2.6$ ) and $\sigma_{t o t} \operatorname{PDG}([42]$ ) (Fig.4b long-dashed line) show practically the same $s$ dependence.

Other interesting characteristics of the diffraction minimum are the height of the dip, which is reflected in the difference between the sizes of the minimum and the second maximum of the differential cross sections, and the 
Table 3: The energy dependence of the characteristics of the dip of $d \sigma / d t$

\begin{tabular}{|c|c|c|c|c|c|c|}
\hline$\sqrt{s}, \mathrm{GeV}$ & $R_{\max / \min }$ & $W_{1 / 2 h}, \mathrm{GeV}^{2}$ & & $\sqrt{s}, \mathrm{TeV}$ & $R_{\max / \min }$ & $W_{1 / 2 h}, \mathrm{GeV}^{2}$ \\
\hline 13. & 1.024 & 0.1544 & 0.1 & 1.49 & 0.173 \\
15. & 1.22 & 0.14605 & 0.2 & 1.23 & 0.152 \\
18. & 1.851 & 0.237 & 0.5 & 1.19 & 0.138 \\
20. & 2.85 & 0.2486 & 0.7 & 1.19 & 0.1375 \\
25 & 5.22 & 0.258 & 1.0 & 1.22 & 0.117 \\
30. & 7.32 & 0.257 & 2.0 & 1.285 & 0.111 \\
32. & 7.12 & 0.255 & 4.0 & 1.37 & 0.105 \\
35. & 6.23 & 0.267 & 7.0 & 1.45 & 0.10 \\
40. & 4.66 & 0.246 & 8.0 & 1.49 & 0.098 \\
50. & 2.98 & 0.2335 & 13.0 & 1.6 & 0.097 \\
60. & 1.64 & 0.224 & & 20.0 & 1.7 & 0.088 \\
\hline
\end{tabular}

width of the diffraction dip at half its height. These values are determined on the one hand, by the slope of the imaginary part in this domain and, on the other hand, by the contributions and $t$ and $s$ dependence of the real part of the spin-non-flip scattering amplitude and by the contribution of the spin-flip scattering amplitude. The depth of the dip can be represented as relations between the maximum and minimum of the dip $-R(s)=\frac{d \sigma / d t_{\max }}{d \sigma / d t_{\min }}$. The energy dependence of these values is represented in Table 3 . We can see that $R_{\max / \min }$ grows faster at low energies and reaches the maximum in the domain around $\sqrt{s}=30 \mathrm{GeV}$ where the diffraction dip has a maximum value. It reflects that the real part of the spin-non-flip amplitude in the elastic proton-proton scattering changes its sign at $t=0$. As was noted above, it is a remarkable fact that the size of the real part in the dip region hardly correlates with the size of the real part at $t=0$. This takes place also for proton-antiproton scattering. The real part in that case changes its sign at $t=0$ approximately around $\sqrt{s}=13 \mathrm{GeV}$. And the diffraction dip in $p \bar{p}$ scattering also has its maximal value at that energy.

At higher energy, we can see from Table 3 that $R_{\max / \min }$ reaches its minimal value at $\sqrt{s}=500-700 \mathrm{GeV}$. It means that the real part of the scattering amplitude relative to the imaginary part has a maximal value in this energy region. At higher energy $R_{\max / \min }$ is increasing weakly with respect to the slowly decreasing value of $\rho(s, t=0)$. The inverse value of $R_{i n v}$ in some approximation shows the ratio of the real to imaginary part of 

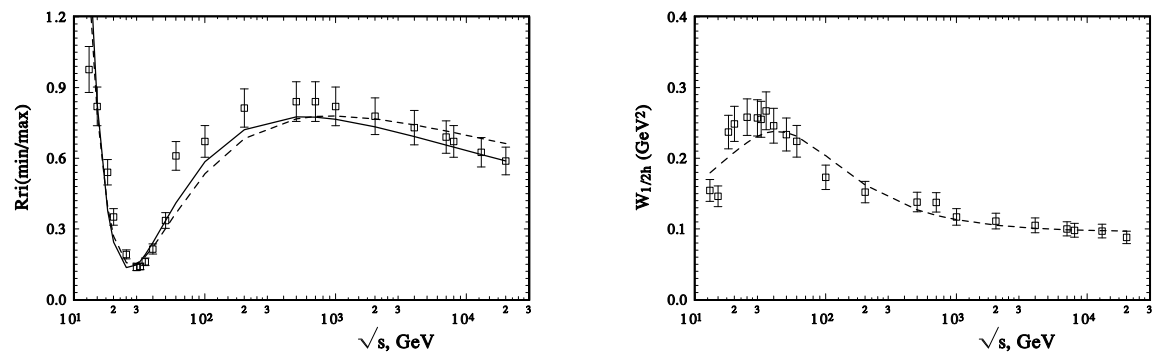

Figure 5: [left] The energy dependence of - $R_{i n v}$ (points - the HEGS model calculations; the hard line the approximation by eq. (13) with $n=2$, dashed line the approximation by eq. (13) with $n=2.6$ ); [right] the energy dependence of the wide of the diffraction dip $W_{1 / 2 h}(s)$ squares - from the HEGS model calculations; dashed line (the approximations by eq.(14)).

the scattering amplitude in this domain of $t$. It can be approximated

$$
R_{\text {inv }}=a_{1}\left(a_{3}+\left(a_{2}-1 / \ln \left(s / s_{0}\right)\right)^{2} / \ln \left(s / s_{0}\right)^{2}\right) .
$$

with $a_{1}=1.07510^{4} \pm 350, a_{2}=0.153 \pm 0.001, a_{3}=3.610^{-4} \pm 210^{-5}$, $s_{0}=1 \mathrm{GeV}^{2}, n=1.732 \pm 0.015$ with $\sum \chi_{i}^{2}=65$; and $a_{1}=809 \pm 120$, $a_{2}=0.306 \pm 0.002, a_{3}=1.4410^{-3} \pm 210^{-4}, s_{0}=1 \mathrm{GeV}^{2}, n=2$ with $\sum \chi_{i}^{2}=80$. The energy dependence is shown in Fig.5a with $n=2$ and $n=1.732$.

Another characteristic of the form of the dip of the differential cross sections is the value of $W_{\frac{1}{2} h}=t_{R}-t_{L}, \mathrm{GeV}^{2}$ - the width of the dip at half its height. Again, this value grows at low energies and after $\sqrt{s}=30 \mathrm{GeV}$ slowly decreases. However, the width decreases faster than $R_{i n v}$. When it changes two times (in the domain $0.1 \leq \sqrt{s} \leq 20 \mathrm{TeV}$ ), the $R_{i n v}$ changes on $14 \%$ only. It can be represented in the form

$$
W_{1 / 2 h}=a_{1}+a_{2} /\left(a_{3} / \sqrt{s / s_{0}}+\sqrt{s / s_{0}}\right)
$$

with $a_{1}=0.098 \pm 0.004, a_{2}=12.18 \pm 1.65, a_{3}=1811 \pm 370, s_{0}=1 \mathrm{GeV}^{2}$. The results of the model calculation and the approximation by eq.(14) are shown in Fig.5b. This value essentially changes in the region where $\rho(s, t=0)$ changes its sign. At large energies it has small changes.

From a more profound analysis the slope of the real part in the dip region and some possible contribution of the spin-flip amplitude can be obtained - However, it requires high precision data in the dip region and a more complicated special work. 


\section{Conclusions}

The form and energy dependence of the diffraction minimum of the differential cross sections of the elastic hadron-hadron scattering gave the valuable information about the structure of the hadron scattering amplitude and hence about the dynamics of strong interactions. The diffraction minimum corresponds to the change of the sign of the imaginary part of the spinnon-flip hadronic scattering amplitude and is created under a strong impact of the unitarization procedure. Its dip depends on the contributions of the real part of the spin-non-flip amplitude and the whole contribution of the spin-flip scattering amplitude. The HEGS model reproduces well the form and the energy dependence of the diffraction dip of the proton-proton and proton antiproton elastic scattering. The predictions of the model in most part reproduce the form of the differential cross section at $\sqrt{s}=13 \mathrm{TeV}$. It means that the energy dependence of the scattering amplitude determined in the HEGS model and unitarization procedure in the form of the standard eikonal representation satisfies the experimental data in the huge energy region (from $\sqrt{s}=9 \mathrm{GeV}$ up to $\sqrt{s}=13 \mathrm{TeV}$ ). It should be noted that the real part of the scattering amplitude, on which the form and energy dependence of the diffraction dip heavily depend, is determined in the framework of the HEGS model only by the complex $\hat{s}$, and hence it is tightly connected with the imaginary part of the scattering amplitude and satisfies the analyticity and the dispersion relations. It allows one to determined the energy dependence of the main characteristic of the diffraction dip - the position of the minimum $t_{\text {min }}(s)$, its width $W_{1 / 2 h}(s)$ at half of the difference between the maximum and minimum of the dip, and the ratio of the maximum to minimum of the diffraction $\operatorname{dip} R_{\max / \min }(s)$ and its inverse value $R_{\text {inv }}(s)$. These characteristics are important for the analysis of the structure of the elastic scattering amplitude in the domain of the diffraction dip. There is a remarkable fact that the size of the real part in the dip region hardly correlates with the size of the real part at $t=0$. We find that the scaling properties are performed on the average. Really, the energy dependence of the product $t_{\text {min }}(s) \sigma_{\text {tot }}(s)$ has a complicated form. The calculations in the framework of the HEGS model and with and energy independence of the product of $t_{\min }$ (eq.(11) with $n=2.6$ ) and $\sigma_{\text {tot }} \mathrm{PDG}([42]$ ) (Fig.4b long-dashed line) show practically the same $s$ dependence. It is shown that the width of the dip of the differential cross sections decreases faster than $R_{i n v}$. Of course, the

HEGS model is oversimplified, and to reproduce quantitatively the different 
thin structures of the scattering amplitude, a wider analysis is needed. This concerns the fixed intercept taken from the deep inelastic processes and the fixed Regge slope $\alpha^{\prime}$, as well as the form of the spin-flip amplitude. Such analysis requires the use of a wider circle of experimental data, including the polarization data and the normalized new data on the elastic $p p$ scattering at $\sqrt{s}=13 \mathrm{TeV}$.

Acknowledgement: The author would like to thank J. -R. Cudell for helpful discussions, gratefully acknowledges the financial support from FRNS and would like to thank the University of Liège where part of this work was done.

\section{References}

[1] R. Fiore, L. Jenkovszky, R. Orava, E. Predazzi, A. Prokudin, O. Selyugin, Mod.Phys., A24 (2009) 2551.

[2] B.Z. Kopeliovich and B.G. Zakharov, Phys.lett. B 226 (1989) 156.

[3] S.V. Goloskokov, S.P. Kuleshov, O.V. Selyugin, Z.Phys. C 50 (1991) 455.

[4] M. Anselmino and S. Forte, Phys. Rev. Lett. 71 (1993).

[5] A.E. Dorokhov, N.I. Kochelev and Yu.A. Zubov, Int. Jour. Mod. Phys. A 8 (1993) 603.

[6] N. Akchurin, S.V. Goloskokov, O.V. Selyugin, Int. J. Mod. Phys. A 14 (1999) 252.

[7] A.Donnachie, P.V. Landshoff, Phys.Lett., B 727 (2013) 500; erratum Phys.Lett., B 750669.

[8] O.V. Selyugin, Eur.Phys.J. C72 (2012) 2073.

[9] O.V. Selyugin, Phys. Rev. D 91 (2015) 11303.

[10] N.N. Khuri, Proc. of Les Rencontre de Physique de la Villee d'Aoste: Results and perspectives in Particle Physics (M. Greeco ed.), p.701, France (1994).

[11] C. Bourrely et.all.,Proceedings EDS 2005, Blois, France (2005). 
[12] J. R. Cudell, et al. [COMPETE Collaboration], Phys.Rev. D65 (2002) 074024 .

[13] J. R. Cudell, et al. [COMPETE Collaboration], Phys.Rev.Lett. 89, (2002) 201801.

[14] O. Selyugin, Sov. J. Nucl. Phys., 55 (1992) 466.

[15] D. Gross, et al., Phys.Rev.Lett. 41 (1978) 217.

[16] A.A. Kuznetzov et al., preprint JINR P1-80-376, Dubna (1980).

[17] L.A. Fajardo et al., Phys.Rev. D 24 (1981) 46.

[18] J. R. Cudell and O. V. Selyugin, Phys. Rev. Lett. 102 (2009) 032003; arXiv:0812.1892 [hep-ph]].

[19] O.V. Selyugin, J.-R. Cudell, E. Predazzi, Eur.Phys.J.ST, 162 (2008) 37.

[20] O. Selyugin, Phys. Lett. B 333 (1994) 245.

[21] N. H. Buttimore, E. Gotsman, E. Leader, Phys. Rev. D 35 (1987) 407.

[22] O. V. Selyugin, Phys. Rev. D 60 (1999) 074028.

[23] X.D. Ji, Phys. Lett., 78B, 610 (1997); Phys. Rev. D 55, 7114 (1997).

[24] O.V. Selyugin and O.V. Teryaev, Phys.Rev. D 79, 033003 (2009).

[25] O.V. Selyugin, Phys. Rev., D 89 (2014) 093007.

[26] O.V. Selyugin, J.-R. Cudell, talk in the Workshop on Diffraction in High-Energy Physics, Aciriale (Italy), September 3-8 (2016),

[27] M.V. Galynskii, E.A. Kuraev, Phys.Rev., D 89 (2014) 054005.

[28] O. V. Selyugin, PEPAN Letters, 13 (2016) 324.

[29] http://durpdg.dur.ac.uk/hepdata/reac.html.

[30] K.R. Schubert, In Landolt-Bronstein, New Series, v. 1/9a, (1979).

[31] The TOTEM Collaboration (G. Antchev et al.) Nucl. Phys. B 899 (2015) 527. 
[32] G. Antchev et al. (TOTEM Coll.) Eur.Phys.Lett., 96 (2011) 21002.

[33] G. Antchev et all. (TOTEM Coll.) Eur.Phys.Lett. 101 (2013) 21002.

[34] G. Antchev et all. (TOTEM Coll.), CERN-PH-EP-2015-325.

[35] The ATTLAS Collaboration, Nucl. Phys. B 889 (2002) 486.

[36] The ATTLAS Collaboration . Nucl.Phys., B 889 (2014) 486.

[37] F. Nemes (TOTEM Collaboration), talk in the Workshop on Diffraction in High-Energy Physics, Aciriale (Italy), September 3-8 (2016),

[38] J. Dias de Deus, Nucl.Phys. B 59 (1973) 231.

[39] G. Alberi and G. Goggi, Phys.Rep. 74 (1981) 1.

[40] R. Henzi, Nucl.phys. , B 104 (1976) 52.

[41] T. Csorgo, R.J. Glauber, and F. Nemes, arXiv:1311.2308

[42] PDG Collaboration, China Phys. C38 (2014) 090513. 